\title{
Where latest advances in HIV are shared: 21st Bangkok International Symposium on HIV Medicine
}

\author{
Pirapon June Ohata*,1, Win Min Han ${ }^{1}$, Donn Colby², Stephen Kerr ${ }^{1,3}$, Anchalee \\ Avihingsanon ${ }^{1,3}$, Thanyawee Puthanakit ${ }^{1,4}$, Nittaya Phanuphak ${ }^{2,5}$, Pich Seekaew ${ }^{5}$, \\ Sasiwimol Ubolyam ${ }^{1}$, Sivaporn Gatechompol ${ }^{1,3}$, Eugene Kroon ${ }^{2}$, Kesdao Nanthapisal ${ }^{1}$, \\ Chowalit Phadungphon ${ }^{1}$, Pornwinit Sattayamong ${ }^{1}$ \& Praphan Phanuphak ${ }^{1,2,5}$ \\ ${ }^{1}$ HIV-NAT, Thai Red Cross AIDS Research Centre, 104 Ratchadamri Rd, Pathumwan, Bangkok 10330, Thailand \\ ${ }^{2}$ SEARCH, Thai Red Cross AIDS Research Centre, 104 Ratchadamri Rd, Pathumwan, Bangkok 10330, Thailand \\ ${ }^{3}$ Department of Medicine, Faculty of Medicine, Chulalongkorn University, Rama IV Rd, Pathumwan, Bangkok 10330, Thailand \\ ${ }^{4}$ Department of Pediatrics, Faculty of Medicine, Chulalongkorn University, Rama IV Rd, Pathumwan, Bangkok 10330, Thailand \\ ${ }^{5}$ Prevention Unit, Thai Red Cross AIDS Research Centre, 104 Ratchadamri Rd, Pathumwan, Bangkok 10330, Thailand \\ *Author for correspondence: Tel.: +662 652 3040; Fax: +662 252 5779; juneohata4@gmail.com
}

Bangkok International Symposium on HIV Medicine is the largest regional conference on clinical HIV medicine in Southeast Asia. Held annually during the third week of January and spanning 3 days, the symposium provides updates on HIV and co-infection treatment and prevention. It is sponsored by HIV Netherlands Australia Thailand Research Collaboration (HIV-NAT), the first and largest clinical research center in Thailand and region. HIV-NAT was founded by the late David Cooper, late Joep Lange and Emeritus Praphan Phanuphak, who dedicated their lives to making HIV care and treatment accessible to people living with HIV in Thailand and throughout the developing world. The symposium continues its tradition of bringing the latest information in the field of HIV medicine to healthcare professionals.

First draft submitted: 28 January 2019; Accepted for publication: 29 January 2019; Published online: 4 March 2019

\section{Special lecture: Professor David Cooper Memorial Lecture (antiretroviral therapy)}

Antiretroviral therapy: advances \& impact

Peter Reiss (Department of Global Health, Academic Medical Center, and Amsterdam Institute of Global Health and Development [AIGHD], Amsterdam, The Netherlands) opened the first Memorial Lecture as his personal tribute to the late Dr Cooper as a key architect of the 'antiretroviral treatment odyssey'. He shared dozens of antiretroviral (ARV)-randomized clinical trials (RCT) that had been conducted by Dr Cooper and colleagues over the past three decades. Dr Cooper and colleagues were among the first researchers to report their clinical observation in people living with human immunodeficiency virus (PLHIV) of body fat changes/lipodystrophy syndrome. He then shared stories of how HIV Netherlands Australia Thailand Research Collaboration (HIV-NAT) was established and its vision to move HIV-related clinical research to Thailand and other resource limited settings (RLS). HIVNAT has become a world-renowned research center and many of its studies [1-3] have impacted multiple regional and international guidelines. Many challenges remain globally, including antiretroviral therapy (ART) scale up and the necessity to improve the care cascade for the intersecting syndemics of communicable and noncommunicable diseases.

\section{Special lecture: Professor Joep Lange \& Ms Jacqueline van Tongeren Memorial Lecture (access to care)}

How close are we to treatment for all?

Andrew Hill (Pharmacology Department, University of Liverpool, Liverpool, UK) opened the second Memorial Lecture by giving an overview of the global HIV epidemic [4]. Although there are a range of countries that have reached Joint United Nations Programme on HIV and AIDS (UNAIDS) 90-90-90 targets (90\% of people have been tested, 90\% of diagnosed PLHIV on ART and 90\% of PLHIV on ART have viral suppression), there are

Future 8 Medicine 
still gaps existing among many settings to reach those targets. The countries with the highest prevalence rates and highest number of PLHIV are actually doing well at getting ART to the people who need it and decreasing the numbers of new HIV infections. In 2019 and in the near future, majority of new HIV infections will occur in countries with low HIV prevalence $(<4.5 \%)$; there will be larger populations and higher rates of new HIV infections and related deaths. Many of these countries are also affected by civil unrest, military conflict or repression of human rights, which all impede the fight against HIV. It is these countries which need more funding and help if an end to AIDS is ever to be achieved. He also stressed that global ART treatment demand will continue to rise unless HIV infection rates fall, stressing the importance of a combination of high treatment coverage with biomedical prevention strategies such as pre-exposure prophylaxis (PrEP) to control HIV epidemics. He ended his talk by acknowledging Dr Lange and Dr Cooper for their dedication to PLHIV since the 1980s.

\section{Overview of tuberculosis treatment}

Anton Pozniak (Chelsea \& Westminster Hospital Foundation Trust, London, UK) provided an overview of the intersection between tuberculosis (TB) and HIV. He covered TB therapy in HIV-infected patients, when and what TB medications should be started, TB and ARV drug-drug interactions, immune reconstitution inflammatory syndrome (IRIS) and management of multidrug-resistant (MDR)/extensively drug-resistant (XDR) TB. He emphasized that TB treatment should be started immediately, followed by early ART [5-8]. He talked at length about rifampicin and rifabutin, and how it interacts with ART (i.e., protease inhibitors boosted with ritonavir [PI/r] [9], efavirenz [EFV] [10-12], integrase inhibitors [INSTIS], nucleoside/nucleotide reverse transcriptase inhibitors [NRTIs], particularly tenofovir alafenamide [TAF]). Regarding IRIS, prednisolone, added to TB treatment, could prevent paradoxical TB IRIS from occurring [13]. The treatment for MDR-TB was discussed, including drug-drug interactions of MDR/extensively drug-resistant TB drugs and ARVs. The 2018 global new TB drug pipeline includes several new drugs under study, but the high costs of new drugs will limit their availability and accessibility in low- and middle-income countries.

\section{Sexually transmitted infections among men who have sex with men \& transgendered populations} Kenneth Mayer (The Fenway Institute, Fenway Health, Boston, MA, USA) stated that the prevalence of sexually transmitted infections (STIs) is increasing globally, especially among men who have sex with men (MSM). Biology, behavior and epidemiology can increase the risk of HIV acquisition in the presence of STI, often referred to as 'syndemics' because of their synergistic relationship. PrEP and treatment as prevention (TasP) can reduce the spread of HIV, but do not prevent an increased spread of STI [14]. Discrimination in healthcare settings is very common, especially for transgender individuals; this significantly impacts the access to and uptake of testing for STIs.

\section{Best approach to end mother-to-child transmission}

Thanyawee Puthanakit (HIV-NAT, Thai Red Cross AIDS Research Centre and Department of Pediatrics, Faculty of Medicine, Chulalongkorn University, Bangkok, Thailand) stated that the first thing is to define the goal and then identify the gaps. She showed the successes from USA, Thailand and Malaysia in achieving $<2 \%$ transmission rate. The most important thing in the prevention of mother-to-child transmission (PMTCT) is to diagnose HIV. She went over the US Department of Health and Human Services (December 2018), WHO (December 2018) and the Thai (2017) guidelines for the treatment of pregnant women, and the service delivery model for PMTCT from Thailand. Recent data from Namibia on the use of dolutegravir (DTG) during first trimester pregnancy and the reported increased risk for neural tube defects was present. All guidelines now caution against the use of DTG during early pregnancy, but more data is needed and is expected in 2019, to confirm the risk. Also discussed were raltegravir (RAL) intensification during pregnancy [15], the WHO guidelines supporting PrEP for pregnant women and neonatal post-exposure prophylaxis (PEP). Stigma continues to be a challenge but ending MTCT can be achieved.

\section{What have we learned from acute HIV research to guide prevention \& care?}

Jintanat Ananworanich (US Military HIV Research Program, MD, USA) said that acute HIV infection is a short but critical period, during the first 6 weeks of infection. This short but dynamic period sets the stage for long-term clinical and treatment outcomes. We have a huge opportunity to learn from acute HIV research to inform us for prevention and treatment, and Thailand has been at the forefront of this research through the efforts of Thai Red Cross AIDS Research Centre. She shared her lessons learned from SEARCH 010/RV254 cohort of acute HIV 
infection, which now has over 580 participants and is among the largest cohorts of its kind in the world. The acute HIV diagnosis algorithm implemented at the Thai Red Cross Anonymous clinic has screened over 300,000 people since 2009, to detect over 700 acute HIV infections. Models show that detection of acute HIV infection and early initiation of ART can prevent further HIV transmission. Benefits of implementing the acute infection cohort in Thailand include data on ARV primary drug resistance [16] and how to test for and diagnose HIV infection early, before the antibody response has fully developed [17]. From the patient perspective, immediate ART was well accepted (99\%). Early ART also was able to normalize CD4/CD8 ratio in acute infection, which is not seen to the same degree in chronic infection. Early ART during acute infection can also preserve the brain [18], the immune system and limit viral diversity. However, even very early ART cannot prevent seeding of the viral reservoir in the lymph nodes and the gut, although there is evidence that the reservoir size may be reduced in very early treated individuals.

\section{The Great Debate: everyone in Southeast Asia should be screened \& treated for latent TB}

A popular session in the symposium is always The Great Debate, in which the audience is polled on a question before and after the debate, and the team that increases its percent of the vote the most is declared the winner. The debate was moderated by Dr Ananworanich. The 'Pro' team was comprised of Anchalee Avihingsanon (HIVNAT, Thai Red Cross AIDS Research Centre) and Frank Cobelens (Amsterdam Institute of Global Health and Development, Amsterdam, The Netherlands). The 'Con' team was comprised of Evy Yunihastuti (Department of Internal Medicine, University of Indonesia, Jakarta, Indonesia) and Dr Pozniak. The 'Pro' team started the debate by asking why people are not debating on whether to treat hypertension or not. Why is management of latent TB infection (LTBI) not approached like hypertension, another chronic condition. There is a guideline on how to manage LTBI treatment so why are physicians not abiding by it? The 'Con' team retorted that the 'Pro' team were just dreamers and that in reality, attempting to provide LTBI treatment to all will not end the TB epidemic. Much work is needed to find index cases so why waste time with LTBI? The 'Pro' team counteracted by saying that the 'Con' team was complacent and not visionary. Elderly, children under 5 years-old and PLHIV were at risk of developing TB so it makes sense to 'nip the disease in its bud'. The 'Con' team ended their argument concluding that people should not be treated for TB without having a confirmed result. The 'Pro' team won the debate, increasing its share of the audience vote from $48 \%$ before the debate to $91 \%$. Now comes the more difficult task of trying to make it happen.

The next symposium, 22nd Bangkok International Symposium on HIV Medicine, will be held on 15-17 January 2020 .

\section{Acknowledgements}

The authors would like to thank all of the speakers for their continued support in providing the latest information on HIV to the symposium, the moderators for coordinating with the speakers for the workshop, the chairpersons for introducing the speakers and making sure that each lecture is presented within the time limit allotted for them.

\section{Financial \& competing interests disclosure}

The 21st Bangkok International Symposium on HIV Medicine was supported by the ViiV Healthcare, Gilead, Mylan Atlanta Medicare, amfAR/TREAT Asia, The Kirby Institute, MSD, Camber pharmaceuticals, Biomedia, Sanofi, Qiagen (Thailand), Government Pharmaceutical Organization (GPO), Pacific Biotech, RND Diagnostics and Thailand Convention and Exhibition Bureau (TCEB). A Avihingsanon participated in a company sponsored speaker's bureau from Jensen-Cilag. The authors have no other affiliations or financial involvement with any organizations or entity with a financial interest in or financial conflict with the subject matter or materials discussed in the manuscript apart from those disclosed.

No writing assistance was utilized in the production of the manuscript.

\section{References}

1 Kroon ED, Ungsedhapand C, Ruxrungtham $\mathrm{K}$ et al. A randomized, double-blind trial of half versus standard dose of zidovudine plus zalcitabine in Thai HIV-1-infected patients (study HIV-NAT 001). HIV Netherlands Australia Thailand Research Collaboration. AIDS 14(10), 1349-1356 (2000).

2 Ruxrungtham K, Kroon ED, Ungsedhapand C et al. A randomized, dose-finding study with didanosine plus stavudine versus didanosine alone in antiviral-naive, HIV-infected Thai patients. AIDS 14(10), 1375-1382 (2000).

3 Bunupuradah T, Kiertiburanakul S, Avihingsanon A et al. Low-dose versus standard-dose ritonavir-boosted atazanavir in virologically suppressed Thai adults with HIV (LASA): a randomised, open-label, non-inferiority trial. Lancet HIV3(8), e343-e350 (2016). 

1482-1491 (2011).

7 Abdool Karim SS, Naidoo K, Grobler A et al. Integration of antiretroviral therapy with tuberculosis treatment. N. Engl. J. Med. 365(16), 1492-1501 (2011).

8 Dean GL, Edwards SG, Ives NJ et al. Treatment of tuberculosis in HIV-infected persons in the era of highly active antiretroviral therapy. AIDS 16(1), 75-83 (2002).

9 Yapa HM, Boffito M, Pozniak A. Critical review: what dose of rifabutin is recommended with antiretroviral therapy? J. Acquir. Immune Defic. Syndr. 72(2), 138-152 (2016).

10 ENCORE1 Study Group. Efficacy of 400mg efavirenz versus standard 600mg dose in HIV-infected, antiretroviral-naive adults (ENCORE1): a randomised, double-blind, placebo-controlled, non-inferiority trial. Lancet 383(9927), 1474-1482 (2014).

11 Luetkemeyer AF, Rosenkranz SL, Lu D et al. Relationship between weight, efavirenz exposure, and virologic suppression in HIV-infected patients on rifampin-based tuberculosis treatment in the AIDS Clinical Trials Group A5221 STRIDE Study. Clin. Infect. Dis. 57(4), 586-593 (2013).

12 Friedland G, Khoo S, Jack C, Lalloo U. Administration of efavirenz ( $600 \mathrm{mg} /$ day) with rifampicin results in highly variable levels but excellent clinical outcomes in patients treated for tuberculosis and HIV. J. Antimicrob. Chemother. 58(6), 1299-1302 (2006).

13 Meintjes G, Stek C, Blumenthal L et al. Prednisone for the prevention of paradoxical tuberculosis-associated IRIS. N. Engl. J. Med. 379(20), 1915-1925 (2018).

14 Katz DA, Dombrowski JC, Bell TR, Kerani RP, Golden MR. HIV incidence among men who have sex with men after diagnosis with sexually transmitted infections. Sex Transm. Dis. 43(4), 249-254 (2016).

15 Puthanakit T, Thepnarong N. J. Virus Erad. 4, 61-65 (2018).

16 WHO. HIV drug resistance report 2017. (2019). www.who.int/hiv/pub/drugresistance/hivdr-report-2017/en/

17 Conference on Retroviruses and Opportunistic Infections (CROI). How low can you go? Diagnosis of acute infection at very low HIV RNA levels. (2019). www.croiconference.org/sessions/how-low-can-you-go-diagnosis-acute-hiv-infection-very-low-hiv-rna-levels

18 Kore I, Ananworanich J, Valcour V et al. Neuropsychological impairment in acute HIV and the effect of immediate antiretroviral therapy. J. Acquir. Immune Defic. Syndr. 70(4), 393-399 (2015). 